\title{
CONDUCTORS AND THE MODULI OF RESIDUAL PERFECTION
}

\author{
JAMES M. BORGER
}

\begin{abstract}
Let $A$ be a complete discrete valuation ring with possibly imperfect residue field. The purpose of this paper is to give a notion of conductor for Galois representations over $A$ that generalizes the classical Artin conductor. The definition rests on two results of perhaps wider interest: there is a moduli space that parametrizes the ways of modifying $A$ so that its residue field is perfect, and any Galois-theoretic object over $A$ can be recovered from its pullback to the (residually perfect) discrete valuation ring corresponding to the generic point of this moduli space.
\end{abstract}

\section{INTRODUCTION}

Let $A$ be a complete discrete valuation ring of residue characteristic $p>0$. If the residue field of $A$ is perfect, there is a satisfactory theory $[17, \mathrm{IV}, \mathrm{VI}]$ of ramification over $A$. For example, let $\rho$ be a Galois representation over $A$, which is a continuous action of an absolute Galois group of the fraction field of $A$ on a finite-rank complex (for now) vector space. Then there is a non-negative integer, the Artin conductor of $\rho$, that measures the extent to which $\rho$ is ramified. If, on the other hand, we allow the residue field of $A$ to be imperfect, ramification over $A$ is still quite mysterious. This prevents us from understanding, say, ramification in codimension one of local systems on arithmetic surfaces.

The work in this paper began with the observation that much about ramification over $A$ can be understood by simply changing base to sufficiently generic extensions with perfect residue field and of relative ramification index one. The first main point (1.4) is that such extensions make up the (perfect-field-valued) points of a natural representable moduli problem. The universal residual perfection $A^{\mathrm{u}}$ of $A$ is the $A$ algebra corresponding to the representing object itself. It is not a discrete valuation ring - but, in a sense, only because its residue ring is not a field (and actually not even noetherian). The generic residual perfection $A^{\mathrm{g}}$ of $A$ is the $A$-algebra corresponding to the fraction field of the representing object. It is a complete discrete valuation ring with perfect residue field. Both $A^{\mathrm{u}}$ and $A^{\mathrm{g}}$ are, of course, unique up to unique isomorphism.

It may be useful to keep a geometric analogue in mind. If we think of complete residually perfect discrete valuation rings as being like germs of curves, then it is natural to regard $A^{\mathrm{u}}$ as the universal jet on $A$ transverse to the maximal ideal and $A^{\mathrm{g}}$ as the generic jet. (It would be interesting to see if the more sophisticated tools from the theory of jet spaces have any relevance here.) 
There is an explicit description (1.14) of these rings in the first section. For example, if $A=\mathbf{F}_{p}(x) \llbracket y \rrbracket$, then we have

$$
\begin{aligned}
A^{\mathrm{u}} \cong \mathbf{F}_{p}(\bar{x})\left[u_{1}, u_{2}, \ldots\right]^{p^{-\infty}} \llbracket y \rrbracket \text { and } \\
A^{\mathrm{g}} \cong \mathbf{F}_{p}\left(\bar{x}, u_{1}, u_{2}, \ldots\right)^{p^{-\infty}} \llbracket y \rrbracket,
\end{aligned}
$$

where the $A$-algebra structures are determined by the data $x \mapsto \bar{x}+u_{1} y+u_{2} y^{2}+\cdots$ and $y \mapsto y$. If $A=\widehat{\mathbf{Z}[x]_{(p)}}$, we have

$$
\begin{aligned}
& A^{\mathrm{u}} \cong W\left(\mathbf{F}_{p}(\bar{x})\left[u_{1}, u_{2}, \ldots\right]^{p^{-\infty}}\right) \text { and } \\
& A^{\mathrm{g}} \cong W\left(\mathbf{F}_{p}\left(\bar{x}, u_{1}, u_{2}, \ldots\right)^{p^{-\infty}}\right),
\end{aligned}
$$

where $W$ denotes the functor of Witt vectors and the $A$-algebra structures are determined by $x \mapsto\left(\bar{x}, u_{1}^{p}, u_{2}^{p^{2}}, \ldots\right)$.

In the second section, I give some properties of $A^{\mathrm{u}}$ and $A^{\mathrm{g}}$. The most important is that the fraction field of $A$ is algebraically closed in the fraction field of $A^{\mathrm{g}}$. And hence the second main point: a Galois representation $\rho$ over $A$ is determined by its pullback $\left.\rho\right|_{A^{\mathrm{g}}}$ to $A^{\mathrm{g}}$. Therefore, any invariant of $\rho$, such as a measure of ramification, can be recovered (in principal) from $\left.\rho\right|_{A^{\mathrm{g}}}$.

Finally, I argue that in defining "non-logarithmic" conductors for Galois representations over $A$, the most simple-minded way of proceeding along these lines is correct: the conductor $\operatorname{ar}(\rho)$ of $\rho$ should be the classical Artin conductor of $\left.\rho\right|_{A^{\mathrm{g}}}$. (Logarithmic conductors are more subtle. See below.) Taking this as the definition, we have the following result.

Theorem. Let $\rho$ and $\rho^{\prime}$ be Galois representations over $A$.

1. $\operatorname{ar}(\rho)$ is a non-negative integer.

2. $\operatorname{ar}\left(\rho \oplus \rho^{\prime}\right)=\operatorname{ar}(\rho)+\operatorname{ar}\left(\rho^{\prime}\right)$

3. If $\rho$ is trivialized by a residually separable extension of $A$, then $\operatorname{ar}(\rho)$ agrees with the classical Artin conductor of $\rho$.

4. $\operatorname{ar}(\rho)$ is zero if and only if $\rho$ is unramified.

5. $\operatorname{ar}(\rho)$ equals the codimension of the subspace of inertia invariants if and only if $\rho$ is tame.

This theorem is an elementary consequence of the basic properties of $A^{\mathrm{g}}$ proved in section 2. The proof of a slightly stronger version is written down in section 3 .

For Galois representations of rank one, Kato 12 has introduced a logarithmic conductor. As first observed in the work 15 of Matsuda (who credits the observation to T. Saito), there is a non-logarithmic variant of Kato's conductor. In a companion paper, I show this non-logarithmic conductor is invariant under pullback to $A^{\mathrm{g}}$ and obtain the following consequence.

Corollary ([4]). Let $\rho$ be a rank-one Galois representation over A. Then the nonlogarithmic Kato conductor of $\rho$ agrees with $\operatorname{ar}(\rho)$.

Kato's original, logarithmic conductor is not, however, always invariant under pullback to $A^{\mathrm{g}}$, and so the naive logarithmic analogue of $\operatorname{ar}(\cdot)$ does not necessarily agree with Kato's conductor. For some brief thoughts on logarithmic conductors for representations of higher rank, see 3.3 .

Abbes and Saito [1] have defined two $\mathbf{Q}_{\geq 0}$-indexed "upper" filtrations of absolute Galois groups of the fraction fields of complete discrete valuation rings with 
arbitrary residue fields. At the end of section 3, I give a definition of such a filtration, which is compatible in the sense of breaks with this paper's conductor. It is tempting to hope it agrees with Abbes and Saito's non-logarithmic filtration (shifted by one).

Boltje-Cram-Snaith [3] and Zhukov [20] also have approaches to non-abelian ramification theory; the relations with them are even more mysterious.

\section{Conventions}

All rings are commutative and contain 1 , and all ring maps preserve 1 . The fraction field of a domain $R$ is denoted $\operatorname{Fr}(R)$.

An extension of a field is a homomorphism to another field. An extension of a discrete valuation ring is an injective local homomorphism to another discrete valuation ring. In both cases, we usually refer to the target of the morphism, rather than the morphism itself, as the extension.

Throughout, $p$ denotes a fixed prime number, and $A$ is a discrete valuation ring, held fixed in each subsection, whose residue field has characteristic $p$. Its fraction field is denoted by $K$. We say such a ring $A$ is of mixed characteristic if $K$ has characteristic 0 , and is of equal characteristic if $K$ has characteristic $p$. Variants of the words residue and generic refer to the residue and fraction fields of $A$. We also use the same words to refer to extensions. For example, we might say $B / A$ is residually purely inseparable or is generically Galois. An extension $B / A$ is unramified (resp. tame) if it is residually separable and its ramification index is one (resp. not divisible by $p$ ). The notations $e_{B / A}, f_{B / A}$, and $f_{B / A}^{\text {sep }}$ denote the ramification index, residue degree, and separable residue degree of the extension $B / A$.

\section{The moduli of Residual PeRfection}

The purpose of this section is to define the category $\mathrm{CRP}_{A}$ of complete residual perfections of $A$, to prove the objects are parametrized by a moduli space, and to give a concrete description of this moduli space. It is common in such matters that it is easier to come up with the proofs than the statements. It is no different in this section, and for this reason, I have left a number of arguments to the reader. Our general reference for categorical terminology will be Mac Lane's book 14.

1.1. An $\mathbf{F}_{p}$-algebra $R$ is perfect if the endomorphism $F: x \mapsto x^{p}$ of $R$ is an isomorphism. The perfection $R^{\mathrm{pf}}=R^{p^{-\infty}}$ of an $\mathbf{F}_{p^{-}}$algebra $R$ is the universal perfect $\mathbf{F}_{p}$-algebra that $R$ maps to. It is the colimit of the iterates of $F$. For any $\mathbf{F}_{p}$-algebra $S$, let PfAlg ${ }_{S}$ be the full subcategory of the category of $S$-algebras whose objects are perfect.

1.2. Let $\mathrm{CRP}_{A}$ be the full subcategory of the category of $A$-algebras consisting of objects $B$ such that $B$ is flat (i.e., $B$ is torsion-free as an $A$-module), $B$ is complete with respect to the ideal $\mathfrak{p}_{A} B$, and $B / \mathfrak{p}_{A} B$ is perfect. (Note that the second condition forces morphisms to be continuous.) For an object $B \in \mathrm{CRP}_{A}$, let $\mathfrak{p}_{B}$ denote the ideal $\mathfrak{p}_{A} B$ (which might not be prime), let $\bar{B}$ denote $B / \mathfrak{p}_{B}$, and if $x$ is an element of $B$, let $\bar{x}$ denote its image in $\bar{B}$. If $f: B \rightarrow B^{\prime}$ is a morphism in $\mathrm{CRP}_{A}$, let $\bar{f}$ denote its reduction $\bar{B} \rightarrow \overline{B^{\prime}}$. Let $s_{B}$ denote the unique multiplicative section [17, II $\S 4$ Prop. 8] of the map $B \rightarrow \bar{B}$. Since $s_{B}(x)$ is the unique lift of $x$ 
that has a $p^{m}$-th root for all integers $m$, every morphism $f: B \rightarrow B^{\prime}$ in $\mathrm{CRP}_{A}$ satisfies

$$
f \circ s_{B}=s_{B^{\prime}} \circ \bar{f} .
$$

1.3. If $\pi \in A$ is a uniformizer and $x$ is an element of an object $B \in \mathrm{CRP}_{A}$, then there are unique elements $x_{0}, x_{1}, \cdots \in \bar{B}$ such that $x=s_{B}\left(x_{0}\right)+s_{B}\left(x_{1}\right) \pi+\cdots$. We call these the coefficients of $x$ (with respect to $\pi$ ). If $f: B \longrightarrow C$ is a morphism in $\mathrm{CRP}_{A}$, then (1.2.1) implies that the coefficients of $f(x)$ are simply the images under $\bar{f}$ of the coefficients of $x$.

1.4. Theorem. The category $\mathrm{CRP}_{A}$ has an initial object $A^{\mathrm{u}}$, and the functor

$$
\mathrm{CRP}_{A} \rightarrow \mathrm{PfAlg}_{\overline{A^{\mathrm{u}}}}, \quad B \mapsto \bar{B}
$$

is an equivalence of categories.

In other words, the functor $\mathrm{CRP}_{A} \rightarrow \mathrm{PfAlg}_{\bar{A}}$ defined by $B \mapsto \bar{B}$ is a representable moduli problem with universal object $A^{\mathrm{u}}$.

As usual, we can prove such a result in an abstract way or in a concrete way. I will give (or sketch) both. The abstract proof consists in showing directly that the functor $\mathrm{CRP}_{A} \rightarrow \mathrm{PfAlg}_{\bar{A}}$ is a cofibration in groupoids (1.5) and then using Freyd's method to construct $A^{\mathrm{u}}$. While appealing, the abstract proof has the big disadvantage that it appears completely unreasonable to hope to use it to prove some things - for example, that $A^{\mathrm{u}}$ is a domain. The concrete proof, on the other hand, consists in simply giving a presentation of $A^{\mathrm{u}}$ in terms of a $p$-basis of $\bar{A}$ lifted to $A$. The problem with this approach is that it depends on choice, and the manner in which the presentation of $A^{\mathrm{u}}$ depends on this choice is not clear. It would be nice to have an explicit description of $A^{\mathrm{u}}$ in terms of familiar canonical constructions.

In any event, nothing that follows the abstract proof actually makes use of it, and the reader so inclined can skip to 1.8 and take the canonical isomorphisms in 1.15 as definitions.

1.5. Proposition. Let $B$ be an object of $\mathrm{CRP}_{A}$, and let $\tilde{C}$ be a perfect $\bar{B}$-algebra. Then there is an object $C$ of $\mathrm{CRP}_{A}$, equipped with a morphism $B \longrightarrow C$ and a morphism $\tilde{C} \longrightarrow \bar{C}$ of $\bar{B}$-algebras, with the property that for every other such object $D$, there is a unique map $f: C \longrightarrow D$ such that the diagram

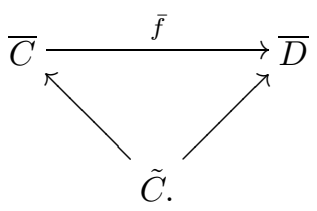

commutes. Moreover, $C$ is unique up to unique isomorphism, and the map $\tilde{C} \rightarrow \bar{C}$ is an isomorphism.

Intuitively, $\tilde{C}$ has a lift to $\mathrm{CRP}_{A}$ that is unique up to unique isomorphism.

Proof. As always, the universality of $C$ will determine it up to unique isomorphism. Let $B[\tilde{C}]$ be the monoid algebra on the multiplicative monoid underlying $\tilde{C}$, and let $C^{\prime}$ be its completion at $\mathfrak{p}_{A} B[\tilde{C}]$. Because $\tilde{C}$ is perfect, $C^{\prime}$ is in $\mathrm{CRP}_{A}$. Now, let $I$ be the kernel of the obvious surjection $\overline{C^{\prime}} \longrightarrow \tilde{C}$, let $J$ be the ideal of $C^{\prime}$ generated by the set $s_{C^{\prime}}(I)$, and put $C=C^{\prime} / J$. The map $\tilde{C} \longrightarrow \bar{C}$ is clearly an isomorphism. The proof that $C$ satisfies the required universal property is left to the reader. 
1.6. Lemma. The category $\mathrm{CRP}_{A}$ has an initial object.

Proof. We apply Freyd's method [14, V.6 Thm. 1]. First, we need to show $\mathrm{CRP}_{A}$ has all (set-indexed) limits. To do this, it is enough to show it has arbitrary (setindexed) products and equalizers of pairs of arrows [14, V.2 Cor. 2].

Fix a uniformizer $\pi$ of $A$.

Let $f, g: B \longrightarrow C$ be two morphisms in $\mathrm{CRP}_{A}$. Let $E \subseteq B$ be their equalizer in the category of rings, and let $\tilde{E}$ be the equalizer of $\bar{f}$ and $\bar{g}$ in the category of rings. Then by 1.3, we see that $E$ consists of the elements whose coefficients all lie in $\tilde{E}$. Therefore, $\mathfrak{p}_{B}^{i} \cap E=\mathfrak{p}_{A}^{i} E$ for all $i$. It follows that $E$ is complete and the natural map $E / p_{A} E \rightarrow \tilde{E}$ is an isomorphism. It is not hard to check $\tilde{E}$ is perfect, and $E$ is clearly torsion-free. Hence $E$ is an object of $\mathrm{CRP}_{A}$.

The reader can check that $\mathrm{CRP}_{A}$ has products.

We will now see that there exists a cardinal number $\kappa$ such that any object $B$ in $\mathrm{CRP}_{A}$ has a subobject whose cardinality is at most $\kappa$. Let $\tilde{C}$ be the perfect subring of $\bar{B}$ generated by the coefficients of the images in $B$ of elements of $A$. Then the cardinality of $\tilde{C}$ is bounded (as $B$ varies). Let $C$ be the closure of the $A$-subalgebra of $B$ generated by the set $s_{B}(\tilde{C})$. Then the cardinalities of such subrings $C$ are also bounded by some cardinal number $\kappa$. It is easy to check that $C / \pi C=\tilde{C}$, and $C$ is clearly complete and flat over $A$. Therefore, $C$ is an object of $\mathrm{CRP}_{A}$.

Let $D$ be the product of all objects $B \in \mathrm{CRP}_{A}$ of cardinality at most $\kappa$. Then by (the proof of) Freyd's theorem [14, V.6 Thm. 1], the equalizer of the set of endomorphisms of $D$ is an initial object.

Proof. (of 1.4) Follows by general reasoning from 1.5 and 1.6 .

1.7. The initial object $A^{\mathrm{u}}$ of $\mathrm{CRP}_{A}$ is called the universal residual perfection of $A$. It is unique up to unique isomorphism. Its reduction $\overline{A^{\mathrm{u}}}$ (or, more properly, the spectrum of its reduction) deserves to be called the moduli space of complete residual perfections of $A$, especially because its points with values in perfect fields correspond to extensions of $A$ of ramification index one:

1.8. Proposition. Let $B$ be an object of $\mathrm{CRP}_{A}$. If $\bar{B}$ is noetherian or a domain, then $B$ is the same. If $\bar{B}$ is a field, then $B$ is a discrete valuation ring.

Proof. Suppose $\bar{B}$ is noetherian. Since $\operatorname{gr}(B)$ is isomorphic to $\bar{B}[X]$, it is noetherian. This implies $B$ is noetherian [2, 10.25].

Now suppose $\bar{B}$ is a domain and $a$ and $b$ are non-zero elements of $B$ with $a b=0$. Since a uniformizer $\pi$ of $A$ is not a zero-divisor in $B$, we can assume $a, b \notin \pi B$, but this immediately contradicts the fact that $\bar{B}$ is a domain.

When $\bar{B}$ is a field, it is easy to see that every element outside $\mathfrak{p}_{B}$ is a unit. Thus $B$ is a noetherian local ring whose maximal ideal is generated by a nonnilpotent element and, hence, a discrete valuation ring [17, I §2].

1.9. Let us now introduce the notation and basic results we will need for the explicit version of 1.4 .

Let $\pi \in A$ be a uniformizer, and let $T$ be a lift to $A$ of a $p$-basis of $\bar{A}$. (A $\operatorname{good}$ general reference for information on $p$-bases is EGA [8, Ch. $0 \S 21]$.) Let $R_{T}$ be the polynomial algebra $\bar{A}\left[u_{t, j} \mid t \in T, j \in \mathbf{Z}_{>0}\right]$. We will see below that $R_{T}^{\mathrm{pf}}$ is naturally $\overline{A^{u}}$, the moduli space we seek. 
1.10. Lemma. Let $Q$ be a residually perfect discrete valuation ring that is a subring of $A$ with the property that $A / Q$ is an extension of ramification index one. Let $B$ be a $Q$-algebra that is complete with respect to an ideal I that contains the image of the maximal ideal of $Q$. Let $n$ be a positive integer, and let $\varphi^{\prime}: A \rightarrow B / I^{n}$ be a $Q$-linear homomorphism. For every $t \in T$, let $x_{t} \in B$ be a lift of $\varphi^{\prime}(t)$. Then there is a unique $Q$-linear map $\varphi: A \rightarrow B$ such that $\varphi^{\prime}=\varphi \bmod I^{n}$ and $\varphi(t)=x_{t}$ for all $t \in T$.

Proof. Since $B$ is complete with respect to $I$, it suffices by induction to prove the existence and uniqueness of $\varphi$ modulo $I^{n+1}$. Let $\Omega_{A / Q}^{1}$ denote the $A$-module of Kähler differentials with respect to $Q$. Since $A / Q$ is formally smooth $[8,19.7 .1]$, some lift $A \rightarrow B / I^{n+1}$ of $\varphi^{\prime}$ exists, and so the set of such lifts is a torsor under

$$
\begin{aligned}
\operatorname{Hom}_{A}\left(\Omega_{A / Q}^{1}, I^{n} / I^{n+1}\right) & =\operatorname{Hom}_{\bar{A}}\left(\bar{A} \otimes_{A} \Omega_{A / Q}^{1}, I^{n} / I^{n+1}\right) \\
& =\operatorname{Hom}_{\bar{A}}\left(\bigoplus_{t \in T} \bar{A} d t, I^{n} / I^{n+1}\right)
\end{aligned}
$$

with the obvious action. It follows that the image of $T$ can be lifted arbitrarily and that any such lift determines $\varphi \bmod I^{n+1}$.

1.11. Construction. Functors $U: \mathrm{CRP}_{A} \rightarrow \operatorname{PfAlg}_{R_{T}^{\mathrm{pf}}}$ and $V: \mathrm{PfAlg}_{R_{T}^{\mathrm{pf}}} \rightarrow \mathrm{CRP}_{A}$.

Let $B$ be an object of $\mathrm{CRP}_{A}$. For $t \in T$ and $j \in \mathbf{Z}_{>0}$, let $v_{t, j} \in \bar{B}$ denote the $j$-th coefficient (1.3) with respect to $\pi$ of the image of $t$ in $B$. The data $u_{t, j} \mapsto v_{t, j}$ gives $\bar{B}$ the structure of an $R_{T}$-algebra. Since $\bar{B}$ is perfect, it has a unique compatible $R_{T}^{\mathrm{pf}}$ algebra structure; $U(B)$ is then $\bar{B}$ with this $R_{T}^{\mathrm{pf}}$-algebra structure. It is easy to see this is functorial.

Let $S$ be a perfect $R_{T}^{\mathrm{pf}}$-algebra. If $A$ is of equal characteristic, set $V(S)=S \llbracket \tilde{\pi} \rrbracket$, where $\tilde{\pi}$ is a free variable. Then $V(S)$ is an $\mathbf{F}_{p} \llbracket \tilde{\pi} \rrbracket$-algebra, and $\tilde{\pi} \mapsto \pi$ makes $A$ into an $\mathbf{F}_{p} \llbracket \tilde{\pi} \rrbracket$-algebra. By 1.10, there is a unique $\mathbf{F}_{p} \llbracket \tilde{\pi} \rrbracket$-linear map $A \rightarrow V(S)$ such that for all $t \in T$,

$$
t \mapsto \bar{t}+\sum_{j \geq 1} u_{t, j} \tilde{\pi}^{j}
$$

If $A$ is of mixed characteristic, let $C$ be the Cohen subring [6, pp. 82-83] of $A$ determined by $T$. It is a complete discrete valuation ring of absolute ramification index one that contains $T$, and $A / C$ is a finite residually trivial extension. Let $W$ be the ring of Witt vectors [10,0.1] with coefficients in $S$ and let $s_{W}: S \rightarrow W$ denote the Teichmüller section. Again by 1.10 (taking $Q=\mathbf{Z}_{p}$ ), there is a unique map $C[X] \rightarrow W \llbracket \tilde{\pi} \rrbracket$ such that $X \mapsto \tilde{\pi}$ and for all $t \in T$, we have

$$
t \mapsto s_{W}(\bar{t})+\sum_{j \geq 1} s_{W}\left(u_{t, j}\right) \tilde{\pi}^{j} .
$$

View $A$ as a quotient of $C[X]$ using the map $X \mapsto \pi$, and put $V(S)=A \otimes_{C[X]} W \llbracket \tilde{\pi} \rrbracket$.

In either case, it is easy to see that $V$ is a functor from $\operatorname{PfAlg}_{R_{T}^{\text {pf }}}$ to the category of $A$-algebras.

1.12. Proposition. The image of $V$ is in $\mathrm{CRP}_{A}$.

Proof. Let $S$ be a perfect $R_{T}^{\mathrm{pf}}$-algebra. Of the properties $V(S)$ is required to satisfy to be in $\mathrm{CRP}_{A}$, the only one that is not immediately clear is flatness over $A$ in mixed 
characteristic. To show this, it suffices to show the element $\pi \otimes 1=1 \otimes \tilde{\pi}$ is not a zero-divisor in $V(S)$.

Let $g(X)$ denote the Eisenstein polynomial that generates the kernel of the surjection $C[X] \rightarrow A$, and let $h(\tilde{\pi})$ denote its image in $W \llbracket \tilde{\pi} \rrbracket$. We will show $\tilde{\pi}$ is not a zero-divisor in the ring $V(S)=W \llbracket \tilde{\pi} \rrbracket /(h(\tilde{\pi}))$. Let $f_{1}(\tilde{\pi})$ and $f_{2}(\tilde{\pi})$ be elements of $W \llbracket \tilde{\pi} \rrbracket$ such that $\tilde{\pi} f_{2}(\tilde{\pi})=h(\tilde{\pi}) f_{1}(\tilde{\pi})$.

Suppose $f_{1}(0) \neq 0$. Then $h(0)$, which is the image of $g(0)$ under the map $C \rightarrow W$, is a zero-divisor. But since $g(X)$ is an Eisenstein polynomial, this implies $p$ is a zero-divisor in $W$, which is impossible. Therefore, we have $f_{1}(0)=0$ and, hence,

$$
\left.f_{2}(\tilde{\pi})=h(\tilde{\pi}) \frac{f_{1}(\tilde{\pi})}{\tilde{\pi}} \in h(\tilde{\pi})\right) W \llbracket \tilde{\pi} \rrbracket .
$$

Thus $f_{2}(\tilde{\pi})$ reduces to zero in $V(S)$, and so $\tilde{\pi}$ is not a zero-divisor in $V(S)$.

1.13. Construction. Natural transformations $\eta: 1 \rightarrow U V$ and $\varepsilon: V U \rightarrow 1$

Let $B$ be an object of $\mathrm{CRP}_{A}$ and $S$ be an object of $\operatorname{PfAlg}_{R_{T}^{\text {pf }}}$. Let $\eta(S)$ be the map

$$
S \longrightarrow U V(S), \quad x \mapsto \overline{s_{V(S)}(x)}
$$

If $A$ is of equal characteristic, let $\varepsilon(B)$ be the composite $V U(B)=\bar{B} \llbracket \tilde{\pi} \rrbracket \longrightarrow B$ defined by $\tilde{\pi} \mapsto \pi$ and $b \mapsto s_{B}(b)$ for $b \in \bar{B}$. It is a homomorphism of rings because $s_{B}$ is [17, II Prop. 8].

If $A$ is of mixed characteristic, there is a unique map $W(\bar{B}) \longrightarrow B$ that reduces to the identity [17, II Prop. 10]. Then, $\tilde{\pi} \mapsto \pi$ determines a map $W(\bar{B}) \llbracket \tilde{\pi} \rrbracket \longrightarrow B$ and, hence, a map

$$
\varepsilon(B): V U(B)=V(\bar{B})=A \otimes_{C[X]} W(\bar{B}) \llbracket \tilde{\pi} \rrbracket \longrightarrow B .
$$

It is easy to see $\varepsilon$ is natural in $B$ and $\eta$ is natural in $S$.

1.14. Theorem. $\langle V, U ; \eta, \varepsilon\rangle$ is an adjoint equivalence between $\mathrm{CRP}_{A}$ and $\mathrm{PfAlg}_{R_{T}^{\mathrm{pf}}}$.

The proof is nothing more than a straight-forward verification of the so-called triangular identities $(\varepsilon V \circ V \eta=1$ and $U \varepsilon \circ \eta U=1)$ and is left to the reader.

1.15. It follows that the unique morphism $A^{\mathrm{u}} \rightarrow V\left(R_{T}^{\mathrm{pf}}\right)$ is an isomorphism. This, in turn, induces a canonical isomorphism $\overline{A^{\mathrm{u}}}=R_{T}^{\mathrm{pf}}$, and so $A^{\mathrm{u}}$ is, by 1.8, a domain. ( $A^{\mathrm{u}}$ is also integrally closed, but we will not use this fact here.) The generic residual perfection $A^{\mathrm{g}}$ of $A$ is the object corresponding under 1.4 to the fraction field of $\overline{A^{\mathrm{u}}}$, that is, the object of $\mathrm{CRP}_{A}$ corresponding to the generic point of the moduli space. It is canonically isomorphic to $V\left(\operatorname{Fr}\left(R_{T}^{\mathrm{pf}}\right)\right)$. By 1.8 , it is a complete discrete valuation ring with perfect residue field.

1.16. Note that if $B$ is an extension of $A$ of ramification index one, then $\mathrm{CRP}_{B}$ is a subcategory of $\mathrm{CRP}_{A}$, and so there is a unique map $A^{\mathrm{u}} \rightarrow B^{\mathrm{u}}$. If it happens that $T$ can be extended to a lift $T^{\prime} \subset B$ of a $p$-basis for $\bar{B}$, this map is the same as the map associated by 1.14 to the inclusion $T \rightarrow T^{\prime}$. The functoriality of the generic residual perfection is a little more subtle. We will consider it in the next section. 
1.17. It is not hard to show that the filtration

$$
F_{n} R_{T}^{\mathrm{pf}}=\bar{A}\left[u_{t, j} \mid t \in T, 1 \leq j \leq n\right]^{\mathrm{pf}}
$$

of $R_{T}$ is independent of the choices of $T$ and $\pi$. Though its role in this paper is small, this filtration is important and should not be ignored. (See 3.3.)

1.18. Remark. I have written this section in the context needed for this paper, but I believe similar constructions could be made in much greater generality.

For example, it appears that the functor $V$ could be defined for rings $A$ (or schemes, or ... ) much more general than discrete valuation rings. It is not yet clear, however, what category should replace $\mathrm{CRP}_{A}$ and what kind of $\operatorname{rings} A$ should be considered. Also, even if we look only at discrete valuation rings $A$, it is probably more natural, from the abstract perspective, to require that each object of $\mathrm{CRP}_{A}$ be equipped with a multiplicative section from its residue ring (and to require that morphisms respect this structure) than to require that it be complete and its residue ring be perfect.

\section{Properties}

As in the previous section, $A^{\mathrm{u}}$ and $A^{\mathrm{g}}$ will denote the universal and generic residual perfections of $A$, and $K^{\mathrm{g}}$ will denote the fraction field of $A^{\mathrm{g}}$.

2.1. Proposition. Let $B$ be an extension of $A$. If $e_{B / A}=1$ and $B / A$ is residually separable, then there exists a unique map $A^{\mathrm{g}} \longrightarrow B^{\mathrm{g}}$ of $A$-algebras.

Note that we do not require that $B / A$ be residually algebraic, and so $B$ satisfies these assumptions if and only if $B / A$ is formally smooth [8, 19.6.1,19.7.1].

Proof. By 1.4, it is enough to show there is a unique map $\overline{A^{\mathrm{g}}} \longrightarrow \overline{B^{\mathrm{g}}}$ of $\overline{A^{\mathrm{u}}}$ algebras. Since $\overline{A^{\mathrm{g}}}$ is the fraction field of $\overline{A^{\mathrm{u}}}$, there is clearly at most one. To show there is at least one, we just have to check that the map $\overline{A^{\mathrm{u}}} \rightarrow \overline{B^{\mathrm{g}}}$ is an inclusion. Since any $p$-basis for $\bar{A}$ can be extended to one for $\bar{B}$, this follows immediately from 1.14 and 1.16 .

2.2. Proposition. Let $B$ be a finite étale extension of $A$. If $C$ is an object of $\mathrm{CRP}_{A}$, then $C \otimes_{A} B$ is an object of $\mathrm{CRP}_{B}$.

Proof. Since $B \otimes_{A} \bar{C}$ is finite étale over $\bar{C}$, which is perfect, it is perfect (by, for example, [8, 21.1.7]). Since $C \otimes_{A} B$ is a finitely generated free $C$-module, it is complete. Therefore, $C \otimes_{A} B$ is an object of $\mathrm{CRP}_{B}$.

2.3. Proposition. Let $B$ be a finite étale extension of $A$. Then the induced maps

$$
B \otimes_{A} A^{\mathrm{u}} \rightarrow B^{\mathrm{u}} \quad \text { and } B \otimes_{A} A^{\mathrm{g}} \rightarrow B^{\mathrm{g}}
$$

of B-algebras are isomorphisms.

Proof. By 2.2, both $B \otimes_{A} A^{\mathrm{u}}$ and $B \otimes_{A} A^{\mathrm{g}}$ are in $\mathrm{CRP}_{B}$, and so by 1.4 , it is enough to show they are isomorphisms after tensoring with $\bar{B}$. Since $\bar{B} / \bar{A}$ is finite and separable, any $p$-basis for $\bar{A}$ is one for $\bar{B}$. Again, 1.14 and 1.16 complete the proof.

The rest of the results in this section are devoted to the proof of the following theorem. 
2.4. Theorem. Fix a separable closure of $K^{\mathrm{g}}$. Then the map $G_{K^{\mathrm{g}}} \rightarrow G_{K}$ of absolute Galois groups is surjective. The induced maps of inertia groups and wild inertia groups are also surjective.

2.5. Remark. It would be interesting to see if the analogous result is true for some motivic Galois group. For example, when $A$ is of equal characteristic, is the functor from crystals (of whatever kind) on $K$ to crystals on $K^{\mathrm{g}}$ fully faithful?

2.6. Lemma. $K$ is algebraically closed in $K^{\mathrm{g}}$.

Proof. If $A$ is residually perfect, then $K^{\mathrm{g}}=K$ and the result is trivially true. Now assume $A$ is not residually perfect. Let $L / K$ be a finite subextension of $K^{\mathrm{g}} / K$, and let $B$ be the normalization of $A$ in $L$. Since $e_{A^{\mathrm{g}} / A}=1$, we have $e_{B / A}=1$. Since $\bar{A}$ is separably closed in $\overline{A^{g}}$, the extension $B / A$ is residually purely inseparable. It is therefore enough to show it is residually separable.

Suppose there is an element $\bar{a} \in \bar{A}-(\bar{A})^{p}$ that has a $p$-th root in $\bar{B}$. We will derive a contradiction (working only modulo $\mathfrak{p}_{A}^{2}$ ). Let $a \in A / \mathfrak{p}_{A}^{2}$ be a lift of $\bar{a}$ and let $x \in B / \mathfrak{p}_{B}^{2}$ be a lift of $\sqrt[p]{\bar{a}}$. Then there is an element $y \in \bar{B}$ such that $a=x^{p}+\pi y$.

Let $\theta$ denote the map $B \longrightarrow A^{\mathrm{g}} / \mathfrak{p}_{A}^{2} A^{\mathrm{g}}$, and let $s$ denote the multiplicative section of the map $A^{\mathrm{g}} / \mathfrak{p}_{A}^{2} A^{\mathrm{g}} \longrightarrow \overline{A^{\mathrm{g}}}$. Since the set $\{\bar{a}\}$ can be extended to a $p$-basis of $\bar{A}$, there is (by, say, 1.14) an element $u \in \overline{A^{\mathrm{g}}}-\bar{A}^{\mathrm{pf}}$ such that $\theta(a)=s(\bar{a})+\pi u$. Take $v \in \overline{A^{\mathrm{g}}}$ such that $\theta(x)=s(\bar{x})+\pi v$. Then we have

$$
\begin{aligned}
s(\bar{a})+\pi u & =\theta(a) \\
& =\theta(x)^{p}+\pi y \\
& =(s(\bar{x})+\pi v)^{p}+\pi y \\
& =s\left(\bar{x}^{p}\right)+\pi y
\end{aligned}
$$

and thus $u=y \in \bar{A}^{\mathrm{pf}}$. Since this cannot be, we have our contradiction.

2.7. An extension $B$ of $A$ is said to be monogenic if there is an element $x \in B$ that generates $B$ as an $A$-algebra. For example, any finite extension that is generically and residually separable is monogenic $[17$, III $\S 6$ Prop. 12].

2.8. Lemma. Let $B$ be a generically separable extension of $A$. Then $B / A$ is monogenic if and only if $B \otimes_{A} A^{\mathrm{g}}$ is a discrete valuation ring. In this case, we have $f_{B \otimes_{A} A^{\mathrm{g}} / A^{\mathrm{g}}}^{\mathrm{sep}}=f_{B / A}^{\mathrm{sep}}$.

Proof. Suppose $B \otimes_{A} A^{\mathrm{g}}$ is a discrete valuation ring. Then we have

$$
A^{\mathrm{g}} \otimes_{A} \Omega_{B / A}^{2}=\Omega_{B \otimes_{A} A^{\mathrm{g}} / A^{\mathrm{g}}}^{2}=0,
$$

where, as usual, $\Omega_{* / *}^{2}$ denotes the second exterior power of the module of relative Kähler differentials. Therefore, $\Omega_{B / A}^{2}$ is zero. The extension $B / A$ is then monogenic by de Smit [7, 4.2].

Now suppose $B / A$ is monogenic. By 2.6, the ring $B \otimes_{A} A^{\mathrm{g}}$ is a domain. By 2.3, it suffices to assume $B / A$ is residually purely inseparable, and so it is enough to show that $B \otimes_{A} A^{\mathrm{g}}$ is generated as an $A^{\mathrm{g}}$-algebra by the root of an Eisenstein polynomial.

There is 5. Prop. 1] an integral extension $A^{\prime}$ of $A$ such that $e_{A^{\prime} / A}=f_{A^{\prime} / A}^{\text {sep }}=1$ and $B \otimes_{A} A^{\prime}$ is a discrete valuation ring with $f_{B \otimes_{A} A^{\prime} / A^{\prime}}=1$. By Zorn's lemma, 
we can assume the residue field of $A^{\prime}$ is $\bar{A}^{\text {pf }}$. Now let $x \in B$ be a generator of the $A$-algebra $B$ and let $f(X) \in A[X]$ be its characteristic polynomial over $A$. Put

$$
y=x \otimes 1-1 \otimes s_{A^{\mathrm{u}}}(\bar{x}) \in B \otimes_{A} A^{\mathrm{u}} .
$$

Then $y$ generates $B \otimes_{A} A^{\mathrm{u}}$ as an $A^{\mathrm{u}}$-algebra, the element $\bar{y} \in \overline{B \otimes_{A} A^{\mathrm{u}}}=\overline{A^{\mathrm{u}}}$ is zero, and the polynomial

$$
g(X)=f\left(X+s_{A^{\mathrm{u}}}(\bar{x})\right) \in A^{\mathrm{u}}[X]
$$

is the characteristic polynomial of $y$ over $A^{\mathrm{u}}$. We will show $\left.g(X)\right|_{A^{\mathrm{g}}}$ is an Eisenstein polynomial.

First, note that since $\bar{y}=0$, we have $g(X) \equiv X^{n} \bmod \mathfrak{p}_{A} A^{\mathrm{u}}$, where $n$ is the degree of $B / A$. All that remains is to show $\left.g(0)\right|_{A^{\mathrm{g}}} \notin \mathfrak{p}_{A^{\mathrm{g}}}^{2}$. Hence, it is enough to show $\left.g(0)\right|_{A^{\mathrm{u}}} \notin \mathfrak{p}_{A}^{2} A^{\mathrm{u}}$ and, therefore, even $\left.g(0)\right|_{A^{\prime}} \notin \mathfrak{p}_{A^{\prime}}^{2}$. But since $\left.y\right|_{A^{\prime}}$ generates $B \otimes_{A} A^{\prime}$ as an $A^{\prime}$-algebra and since $B \otimes_{A} A^{\prime}$ is a discrete valuation ring, $\left.g(X)\right|_{A^{\prime}}$ is an Eisenstein polynomial. Therefore, $\left.g(0)\right|_{A^{\prime}} \notin \mathfrak{p}_{A^{\prime}}^{2}$ and $\left.g(X)\right|_{A^{\mathrm{g}}}$ is an Eisenstein polynomial.

2.9. Lemma. Let $B$ be a finite, generically Galois extension of $A$. Let $B^{\prime}$ be the integral closure of the domain $B \otimes_{A} A^{\mathrm{g}}$. Then $f_{B^{\prime} / A^{\mathrm{g}}}^{\mathrm{sep}}=f_{B / A}^{\mathrm{sep}}$.

Proof. By 2.3, it is enough to assume $B / A$ is residually purely inseparable. Let $G$ be the generic Galois group of $B / A$. Then 2.6 implies $G$ is also the generic Galois group of $B^{\prime} / A^{\mathrm{g}}$. Let $C^{\prime}$ be the maximal étale subextension of $B^{\prime} / A^{\mathrm{g}}$. Then $C^{\prime}$ corresponds to a normal subgroup of $G$ and, hence, to a generically Galois subextension $C$ of $B / A$. Since the extension $B / A$ is residually purely inseparable and $e_{C^{\prime} / A}=1$, the ramification index of $C / A$ is one and its Galois group is a $p$-group.

Now let $D$ be a monogenic subextension of $C / A$. Then $D \otimes_{A} A^{\mathrm{g}}$ is a discrete valuation ring by 2.8. Since $D \otimes_{A} A^{\mathrm{g}} / A^{\mathrm{g}}$ is a subextension of $C^{\prime} / A^{\mathrm{g}}$, it is étale. Now, the residue field of $D \otimes_{A} A^{\mathrm{g}}$ is $\left(\bar{D} \otimes_{\bar{A}} \overline{A^{\mathrm{g}}}\right)_{\text {red }}$. Since $\bar{D}$ is a finite purely inseparable extension of $\bar{A}$, this reduced quotient is $\overline{A^{\mathrm{g}}}$. Thus, the ètale extension $D \otimes_{A} A^{\mathrm{g}} / A^{\mathrm{g}}$ is trivial, and therefore, so is $D / A$.

But since the generic Galois group of $C / A$ is a $p$-group and since all extensions of degree $p$ are monogenic, the only way for all monogenic subextensions of $C / A$ to be trivial is if $C / A$ itself is trivial. And this can happen only if $C^{\prime} / A^{\mathrm{g}}$ is trivial.

Proof. (of 2.4) It follows from 2.6 that $K$ is separably closed in $K^{\mathrm{g}}$. The surjectivity of $G_{K^{\mathrm{g}}} \rightarrow G_{K}$ is just the translation of this into Galois theory.

The image of the inertia subgroup of $G_{K^{\mathrm{g}}}$ is then a closed normal subgroup $N$ of $G_{K}$ and is contained in the inertia subgroup of $G_{K}$. Let $M$ be the corresponding extension of $K$. Now, for any finite extension $B$ of $A$ that is contained in $M$, we see, by lemma 2.9, that $B / A$ is unramified. Therefore, $N$ is the entire inertia group of $K$.

Since the wild inertia groups are the unique (pro-)p-Sylow subgroups of the corresponding inertia groups [19, Ch. I, 1.1], the surjectivity of the map between wild inertia groups follows immediately.

2.10. Questions. The maps $A \rightarrow A^{\mathrm{u}}$ and $A \rightarrow A^{\mathrm{g}}$ are faithfully flat. How can we best understand the descent data? The generic descent data? What can be said about the structure of the exact sequence

$$
1 \rightarrow H \rightarrow G_{K^{\mathrm{g}}} \rightarrow G_{K} \rightarrow 1 ?
$$


Since $A^{\mathrm{g}}$ is residually perfect, the classfield theory of Hazewinkel and Serre [9] gives a description of the abelianization of $G_{K}$. Does this give a classfield theory describing the abelianization of $G_{K}$ ? When $K$ is a higher local field, how does the classfield theory of $K^{\mathrm{g}}$ relate to Kato and Parshin's classfield theory [11, 16] of $K$ ?

\section{Conductors}

3.1. Fix a field $\Lambda$ whose characteristic is not $p$, and let $\rho$ be a Galois representation over $A$, that is, a homomorphism $\rho: G \longrightarrow \operatorname{Aut}(V)$, where $G$ is the Galois group of a finite generically Galois extension $B$ of $A$ and $V$ is a finite-rank $\Lambda$-module. For any $i \in \mathbf{N}$, let $G_{i}$ be the kernel of the map $G \rightarrow \operatorname{Aut}\left(B / \mathfrak{p}_{B}^{i+1}\right)$. Define

$$
\operatorname{ar}_{\mathrm{n}}^{\mathrm{B}}(\rho)=e_{B / A}^{-1} \sum_{i \geq 0}\left|G_{i}\right| \operatorname{codim} V^{G_{i}}
$$

where $|\cdot|$ denotes cardinality and $\operatorname{codim} V^{G_{i}}$ is the codimension of the subspace of $G_{i}$-invariants. We call $\operatorname{ar}_{\mathrm{n}}^{\mathrm{B}}(\rho)$ the naive Artin conductor of $\rho$ with respect to $B$. It is a non-negative rational number. If $B / A$ is residually separable, $\operatorname{then} \operatorname{ar}_{\mathrm{n}}^{\mathrm{B}}(\rho)$ is left unchanged if we replace $B$ with a larger generically Galois and residually separable extension of $A$. In this case, we will use the notation $\operatorname{ar}_{\mathrm{n}}(\rho)$.

Let $L$ denote the fraction field of $B$. Then by 2.4, $L \otimes_{K} K^{\mathrm{g}}$ is a finite Galois extension of $K^{\mathrm{g}}$, and its Galois group is canonically isomorphic to $G$. Let $\left.\rho\right|_{A^{\mathrm{g}}}$ denote the resulting representation of $\operatorname{Gal}\left(L \otimes_{K} K^{\mathrm{g}} / K\right)$. Define the Artin conductor $\operatorname{ar}(\rho)$ of $\rho$ to be $\operatorname{ar}_{\mathrm{n}}\left(\left.\rho\right|_{A^{\mathrm{g}}}\right)$. We have the following slightly stronger version of the theorem in the introduction:

3.2. Theorem. Let $A, \rho, G, V$, and $B$ be as above, and let $\rho^{\prime}$ be another Galois representation over $A$. Then we have the following:

1. $\operatorname{ar}\left(\rho \oplus \rho^{\prime}\right)=\operatorname{ar}(\rho)+\operatorname{ar}\left(\rho^{\prime}\right)$.

2. $\operatorname{ar}(\rho)$ is a non-negative integer.

3. $\operatorname{ar}(\rho)=0$ if and only if $\rho$ is unramified, i.e., $G_{0}$ acts trivially on $V$.

4. If $B / A$ is monogenic, then $\operatorname{ar}(\rho)=\operatorname{ar}_{\mathrm{n}}^{\mathrm{B}}(\rho)$.

5. The following are equivalent:

(a) $\rho$ is tame, i.e., the $p$-Sylow subgroup of $G_{0}$ acts trivially on $V$

(b) $\operatorname{ar}(\rho)=\operatorname{codim}\left(V^{G_{0}}\right)$

(c) $\operatorname{ar}(\rho) \leq \operatorname{codim}\left(V^{G_{0}}\right)$

Proof. Statement 1 follows from the additivity of the naive Artin conductor. Statement 2 follows from the classical Hasse-Arf theorem [17, VI §2] (if the characteristic of $\Lambda$ is not zero, see [18, 19.3]) and the non-negativity of the naive Artin conductor. Statement 3 follows from the statement about inertia groups in 2.4.

Now consider statement 1 . Let $G^{\prime}$ be the generic Galois group of the extension $B \otimes_{A} A^{\mathrm{g}} / A^{\mathrm{g}}$. By 2.8, the tensor product $B \otimes_{A} A^{\mathrm{g}}$ is a discrete valuation ring. A short argument then shows that for all $i \in \mathbf{N}$, we have

$$
G_{i}=G_{e i}^{\prime}=\cdots=G_{e i+e-1}^{\prime},
$$

where $e=e_{B \otimes_{A} A^{g} / B}$. Therefore, $\operatorname{ar}_{\mathrm{n}}^{\mathrm{B}}(\rho)=\operatorname{ar}_{\mathrm{n}}^{\mathrm{B} \otimes_{\mathrm{A}} \mathrm{A}^{\mathrm{g}}}\left(\left.\rho\right|_{A^{\mathrm{g}}}\right)=\operatorname{ar}(\rho)$.

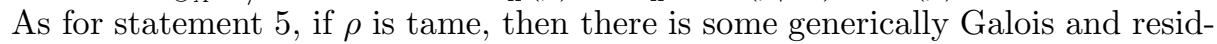
ually separable extension $B$ of $A$ such that $\left.\rho\right|_{B}$ is trivial. Since $B / A$ is residually separable, it is monogenic, and so statement 1 implies $\operatorname{ar}(\rho)=m$. If, on the other hand, we have $\operatorname{ar}(\rho) \leq m$, then $\left.\rho\right|_{A^{\mathrm{g}}}$ is tame, 2.4 therefore implies $\rho$ is tame. 
3.3. Remark. As mentioned in the introduction, logarithmic conductors are more subtle than non-logarithmic ones. For $\chi \in H^{1}(K, \mathbf{Q} / \mathbf{Z})$, let $\operatorname{sw}_{K}(\chi)$ be Kato's Swan conductor [12, 2.3], which equals the logarithmic order of the pole of his refined Swan conductor. Then there can be classes $\chi$ (those that Matsuda says are of type $I I$ [15, 3.2.10]) such that $\operatorname{sw}_{K}(\chi)=\operatorname{sw}_{K}\left(\left.\chi\right|_{A^{g}}\right)+1$. Because of this, the naive Swan analogue of $\operatorname{ar}(\cdot)$ does not always agree with Kato's Swan conductor. I believe that by taking into account the filtration $F_{\bullet} \overline{A^{\mathrm{u}}}$ of $A^{\mathrm{u}}$ (see 1.17), one could give a good definition of a logarithmic conductor. Indeed, using the techniques in Matsuda [4, 15], it is easy to see how to recover the Kato-Swan conductor of $\chi$ from the refined Swan conductor of $\left.\chi\right|_{A^{\mathrm{g}}}$ in equal characteristic.

3.4. Remark. It may also be worth mentioning that the conductor $\operatorname{ar}(\cdot)$ probably does not satisfy an induction formula. Let $A^{\prime} / A$ be a finite generically separable extension, $\rho^{\prime}$ a Galois representation over $A^{\prime}$, and $\rho$ the induced representation over $A$. If $\bar{A}$ is perfect, then [17, VI $\S 2]$

$$
\operatorname{ar}(\rho)=f_{A^{\prime} / A} \operatorname{ar}(\rho)+\operatorname{dim}(\rho) v_{A}(\mathfrak{D}),
$$

where $\mathfrak{D}$ denotes the discriminant of $A^{\prime} / A$. In general, the refined conductor of $\rho$ should be the norm, in some suitable sense, of the refined conductor of $\rho^{\prime}$. When $\bar{A}$ is perfect, the conductor determines the refined conductor up to an element of $A^{*}$, and then 3.4 .1 would follow from such a norm formula for refined conductors. But when $\bar{A}$ is not perfect, the refined conductor contains more information than the conductor together with a unit. In fact, even in the abelian case, the conductor of $\rho^{\prime}$ probably does even determine the conductor of $\rho$ in general.

3.5. There is, however, an apparently satisfactory theory of the upper filtration. In the notation of 3.1, since $G$ is naturally $\operatorname{Gal}\left(L \otimes_{K} K^{\mathrm{g}} / K^{\mathrm{g}}\right)$, it inherits a $\mathbf{Q}_{\geq 0}$-indexed upper filtration [17, IV §3]. This filtration is stable under passage to quotients for the simple reason that the same is true for residually separable extensions. We also have the usual relation to conductors: Following Katz [13, 1.1], there is a break decomposition $V=\oplus_{x} V(x)$, with respect to $G^{\bullet}$, and we have

$$
\operatorname{ar}(\rho)=\operatorname{codim}\left(V^{G_{0}}\right)+\sum_{x \in \mathbf{Q}_{\geq 0}} x \operatorname{dim} V(x)
$$

\section{REFERENCES}

[1] Ahmed Abbes and Takeshi Saito. Ramification of local fields with imperfect residue fields I. To appear.

[2] M. F. Atiyah and I. G. Macdonald. Introduction to commutative algebra. Addison-Wesley Publishing Co., Reading, Mass.-London-Don Mills, Ont., 1969.

[3] Robert Boltje, G.-Martin Cram, and V. P. Snaith. Conductors in the non-separable residue field case. In Algebraic K-theory and algebraic topology (Lake Louise, AB, 1991), pages 1-34. Kluwer Acad. Publ., Dordrecht, 1993.

[4] James Borger. Kato's conductor and generic residual perfection. To appear.

[5] James Borger. A monogenic Hasse-Arf theorem. 1999. To appear in the proceedings of the Luminy conference on ramification theory for arithmetic schemes.

[6] I. S. Cohen. On the structure and ideal theory of complete local rings. Trans. Amer. Math. Soc., 59:54-106, 1946.

[7] Bart de Smit. The different and differentials of local fields with imperfect residue fields. Proc. Edinburgh Math. Soc. (2), 40(2):353-365, 1997.

[8] A. Grothendieck. Éléments de géométrie algébrique. IV. Étude locale des schémas et des morphismes de schémas. I. Inst. Hautes Études Sci. Publ. Math., (20):259, 1964. 
[9] Michiel Hazewinkel. Corps de classes local. In Groupes algébriques. Tome I: Géométrie algébrique, généralités, groupes commutatifs. Masson \& Cie, Éditeur, Paris, 1970. Appendix.

[10] Luc Illusie. Complexe de de Rham-Witt et cohomologie cristalline. Ann. Sci. École Norm. Sup. (4), 12(4):501-661, 1979.

[11] Kazuya Kato. A generalization of local class field theory by using K-groups. I. J. Fac. Sci. Univ. Tokyo Sect. IA Math., 26(2):303-376, 1979.

[12] Kazuya Kato. Swan conductors for characters of degree one in the imperfect residue field case. In Algebraic K-theory and algebraic number theory (Honolulu, HI, 1987), pages 101131. Amer. Math. Soc., Providence, RI, 1989.

[13] Nicholas M. Katz. Gauss sums, Kloosterman sums, and monodromy groups. Princeton University Press, Princeton, NJ, 1988.

[14] Saunders Mac Lane. Categories for the working mathematician. Springer-Verlag, New York, second edition, 1998.

[15] Shigeki Matsuda. On the Swan conductor in positive characteristic. Amer. J. Math., 119(4):705-739, 1997.

[16] A. N. Parshin. Local class field theory. Trudy Mat. Inst. Steklov., 165:143-170, 1984. Algebraic geometry and its applications.

[17] Jean-Pierre Serre. Corps locaux. Hermann, Paris, 1968. Deuxième édition, Publications de l'Université de Nancago, No. VIII.

[18] Jean-Pierre Serre. Représentations linéaires des groupes finis. Hermann, Paris, 1971. Deuxième édition, refondue.

[19] Jean-Pierre Serre. Cohomologie galoisienne. Springer-Verlag, Berlin, fifth edition, 1994.

[20] Igor B. Zhukov. On ramification theory in the imperfect residue field case. Technical report, Nottingham University, May 1998. To appear in the proceedings of the Luminy conference on ramification theory for arithmetic schemes.

University of Chicago, Chicago, Illinois, USA

E-mail address: borger@math.uchicago.edu 